\title{
Phase controlled integrated interferometric single-sideband filter based on planar Bragg gratings implementing photonic Hilbert transform
}

\author{
Chaotan Sima, J. C. Gates, H. L. Rogers, P. L. Mennea, C. Holmes, M. N. Zervas, and P. G.R. Smith \\ Optoelectronics Research Centre, University of Southampton, Southampton SO17 1BJ, United Kingdom
}

\begin{abstract}
The first monolithically integrated all-optical single-sideband filter based on photonic Hilbert transform and planar Bragg gratings is proposed and experimentally demonstrated. Single-sideband suppression of $12 \mathrm{~dB}$ at $6 \mathrm{GHz}$ and sideband switching are achieved via thermal tuning. An X-coupler, photonic Hilbert transformer, flat top reflector and a micro heater are incorporated in a single silicon-on-silica substrate. The device can be thermally tuned by the micro heater on top of the channel waveguide. The device is fabricated using a combination of direct UV grating writing technology and photolithography.
\end{abstract}

Single-sideband (SSB) modulation is a well-known technique in telecommunications for improving performance in terms of required power, enhancing spectral efficiency and reducing fading. In the conventional electronic domain, Hilbert transform techniques have been widely used in SSB modulation applications, and devices have been implemented in both analogue and digital systems and geometries [1]. In the all-optical field, photonic Hilbert transformers (PHTs) offer potential for a wide range of applications as they provide operational bandwidths and speeds far beyond current electronic technologies. In addition they also reduce dispersion related artifacts such as signal power fading [2]. Various methods have been demonstrated to produce PHTs, e.g. in phase-shifted apodized fiber Bragg gratings (FBGs) [2, 3], free space optical components [4], Mach-Zehnder interferometers with optical delay lines $[5,6]$, sampled FBGs [7] and a programmable wave-shaper [8]. Previous works on all-optical SSB modulation such as that reported in [2, 7] deal with complex fiber-based schemes comprising optical circulators, attenuators and optical delay lines. The complexity and size of physically realizing these schemes results in costly systems that lack the mechanical and thermal stability required for system applications.

In this work, we demonstrate a monolithically integrated all-optical SSB filter, for the first time to our knowledge. This initial proof-of-concept device shows $12 \mathrm{~dB}$ suppression ratios at $6 \mathrm{GHz}$ microwave frequency. We use a silica-on-silicon planar format [9], which provides a route to realize compact optical modules for integrated optical applications. This device has the benefits of an integrated single chip, direct thermal tuning, UV phase trimming, fiber compatibility, long term thermal stability and could be used alongside conventional etched silica-on-silicon devices.

The integrated photonic device is composed of an $\mathrm{X}$-coupler [10], a PHT, a flat top reflector and a micro heater, as shown in Fig. 1 [11]. The PHT was implemented using an apodized planar Bragg grating with a $\Pi$ phase shift within the grating [3]. A sincapodized Bragg grating was utilized as the flat top reflector. By applying DC voltages to the miniature nichrome heater, the phase of the optical signal can be finely trimmed through thermal tuning.

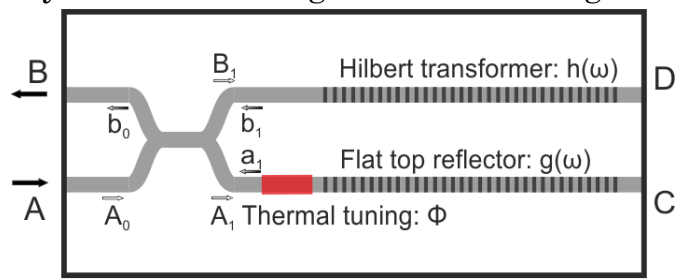

Fig. 1. The principle scheme of the integrated all-optical SSB filter via thermal tuning.

We consider the case in which light is coupled into port $\mathrm{A}$ and received from port $\mathrm{B}$, and assume that both arms have identical waveguide structures. From waveguide coupler theory [12], we get

$$
\begin{gathered}
A_{1}=A_{0} \cos (\kappa \ell) \\
B_{1}=-j A_{0} \sin (\kappa \ell)
\end{gathered}
$$

where $\mathrm{K}$ is the coupling coefficient and $\mathrm{l}$ is the coupling length. After reflection from the gratings, optical fields $a_{1}$ and $b_{1}$ become

$$
\begin{gathered}
a_{1}=A_{1} g(\Delta \omega) e^{j(2 \phi-\beta L)}=A_{0} \cos (\kappa \ell) g(\Delta \omega) e^{j(2 \phi-\beta L)} \\
b_{1}=B_{1} h(\Delta \omega) e^{j(-\beta L)}=-j A_{0} \sin (\kappa \ell) h(\Delta \omega) e^{j(-\beta L)}
\end{gathered}
$$

where $-B \mathrm{~L}$ is the phase shift due to propagation in two arms, $B$ is the wave number and $\Phi$ is the extra phase shift due to thermal tuning. $\Phi=B \Delta \mathrm{L}$ where $\Delta \mathrm{L}$ is the equivalent path length difference. The extra phase shift of light in one round trip is $2 \Phi . h(\Delta \omega)$ and $g(\Delta \omega)$ are reflection coefficients of the PHT and the flat top reflector respectively. $\Delta \omega=\omega^{-} \omega_{0}$ where $\omega$ is the frequency parameter and $\omega_{0}$ is the central frequency. The device output $b_{0}$ is:

$$
b_{0}=-j A_{0} \sin (\kappa \ell) \cos (\kappa \ell) e^{-j \beta L}\left[h(\Delta \omega)+g(\Delta \omega) e^{2 j \phi}\right]
$$


The Hilbert transform grating response function in the frequency domain is [1]:

$$
h(\Delta \omega)=-j \operatorname{sgn}(\Delta \omega) \Pi(\Delta \omega) r_{0}
$$

where $\operatorname{sgn}(\Delta \omega)$ is the sign function (which is +1 for $\Delta \omega>0$ and -1 otherwise), $\Pi(\Delta \omega)$ is the rectangular function (window function), and $r_{0}$ is the maximum reflection coefficient. The PHT has a $\Pi$ phase shift at $\Delta \omega=0$, whereas the amplitude remains constant. For a physically realizable PHT, a practical grating apodization profile has been given in [3]:

$$
\Delta n(z) \propto \frac{\sin ^{2}\left[\pi n_{e f f} \Delta f\left(z-z_{0}\right) / c\right]}{z-z_{0}}
$$

where $\mathrm{n}_{\text {eff }}$ is the grating effective refractive index, $\mathrm{c}$ is the light speed in vacuum, $\mathrm{z}$ is the grating length, $\mathrm{z}_{0}$ is the zero-crossing point in the apodization function, and $\Delta \mathrm{f}$ is the operative bandwidth.

Sinc-apodization of Bragg gratings are used to generate flat top and dispersion-less reflectors [12]. In our case a sinc-apodized grating design is used to obtain similar bandwidth and amplitude responses as our PHT. The grating frequency response is:

$$
g(\Delta \omega)=\Pi(\Delta \omega) r_{0}
$$

The grating apodization profile is given as:

$$
\Delta n(z) \propto \frac{\sin \left[\pi\left(z-z_{0}\right)\right]}{\left(z-z_{0}\right)}
$$

A transfer matrix method was employed for the calculation of the expected device output by calculating the fields in Eq. (5). Figure 2 illustrates the reflectivity spectra of the individual Bragg gratings, the device output (with $\Delta \mathrm{L}$ of $0.2 \mu \mathrm{m}$ and $0.4 \mathrm{\mu m}$ ), and a pseudo color plot of the spectra as a function of $\Delta \mathrm{L}$. Figures $2(\mathrm{a})$ and $2(\mathrm{~b})$ demonstrate the similarity of the sinc-apodized grating response to that of the PHT, except the $\Pi$ phase-shift at the central wavelength. Figure 2(c) shows the resulting
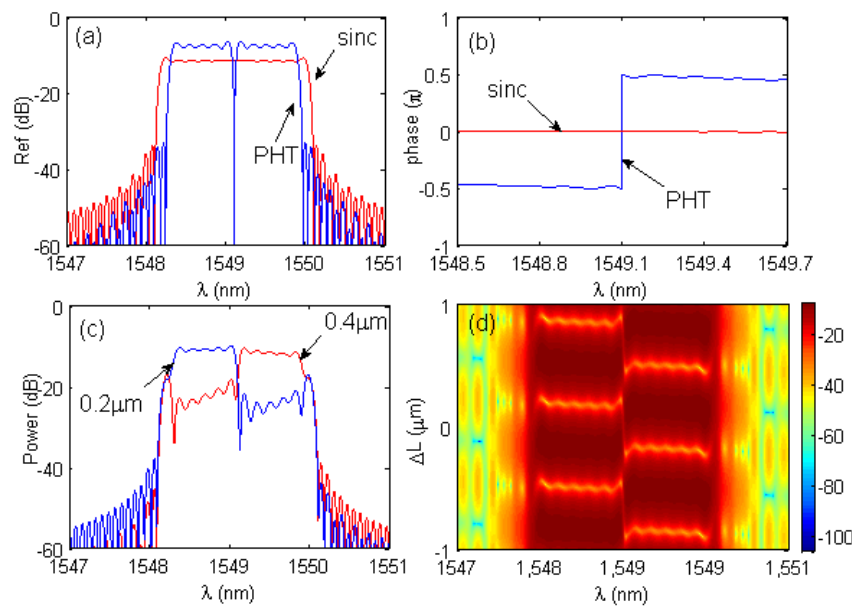

Fig. 2. Simulation: (a) the PHT and sinc-apodized gratings amplitude spectra; (b) normalized gratings phase spectra; (c) device outputs with $\Delta \mathrm{L}$ at $0.2 \mu \mathrm{m}$ (red) and $0.4 \mu \mathrm{m}$ (blue); (d) the intensity color map of the output spectra as a function of $\Delta \mathrm{L}$. interfered output from the device for two optical path difference scenarios, where the opposite side lobes are suppressed. Figure 2(d) presents the effect of optical path difference over an extended range showing a periodic variation.

The proposed planar structures were fabricated using the direct UV gating writing technique [11]. This method involves focusing two crossed laser beams $(\lambda=244 \mathrm{~nm})$ into the photosensitive core of a planar sample. Precise translation of the sample and modulation of the interference pattern creates grating structures and simultaneously defines the channel waveguide.
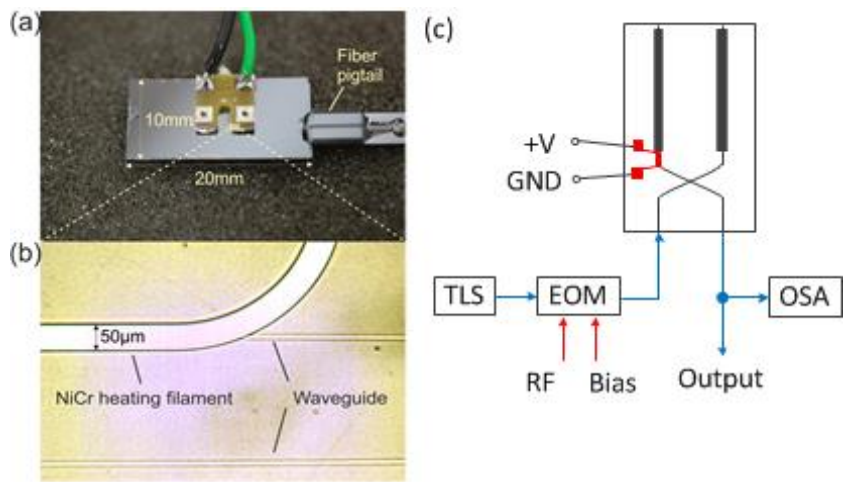

Fig. 3(a) The fabricated device with electro heating elements and a fiber pigtail; (b) the image of Nichrome heater (50 $\mu \mathrm{m}$ wide) on top of the waveguide; (c) the experimental setup of the optical SSB modulation svstem.

The fabricated chip had dimensions of $20 \mathrm{~mm} \times 10$ $\mathrm{mm} \times 1 \mathrm{~mm}$ and a three-layer silica-on-silicon substrate, containing a thermal oxide layer (lower clad), core and an upper clad. The thickness of the core was $\sim 6 \mu \mathrm{m}$, which ensured lateral single mode operation. The two gratings were both $10 \mathrm{~mm}$ long, the effective refractive index of the mode within the gratings was 1.447 , and the maximum refractive index modulation depth was $\Delta \mathrm{n}=0.0003$. The Xcoupler length was $\sim 7.5 \mathrm{~mm}$, with crossing angle 2.5 degrees, yielding $\sim 50: 50$ coupling ratio. The spacing between the X-coupler arms was $250 \mu \mathrm{m}$ to provide coupling with a standard two port fiber v-groove assembly, shown in Fig. 3(a). The fiber v-groove assembly contained two pre-aligned single mode polarization maintaining (PM) fibers and was aligned to the waveguide channels and secured using a UV curing adhesive.

To deliver the thermal energy a $50 \mu \mathrm{m}$-wide by 210 $\mathrm{nm}$-thick nichrome filament was deposited over a 1 $\mathrm{mm}$ length of waveguide, as shown in Fig. 3(b), using photolithography and electron beam metal evaporation. The micro heater's resistance was measured to be $320 \Omega$. The applied DC voltages were tuned between $0 \mathrm{~V}$ and $22 \mathrm{~V}$ by a power supply. The whole device was thermally bonded to a metal block to act as a simple thermal reservoir. The high thermal conductivity of the silicon substrate ensures that the gratings and surrounding waveguides away from the heater are substantially independent of the heater temperature [13]. 
Figure 3(c) shows the experimental setup of the alloptical SSB modulation system. To characterize the sideband suppression the output from a polarized CW tunable laser source (TLS, Agilent 81600B) is sent to a PM electro-optic modulator (EOM) which is amplitude modulated by the RF signal from a vector network analyzer (VNA, Agilent 8753ES). The double sideband (DSB) optical signal from the EOM was launched into port $\mathrm{A}$ of the device and spectral characteristics were measured by an optical spectrum analyzer (OSA) from port B.
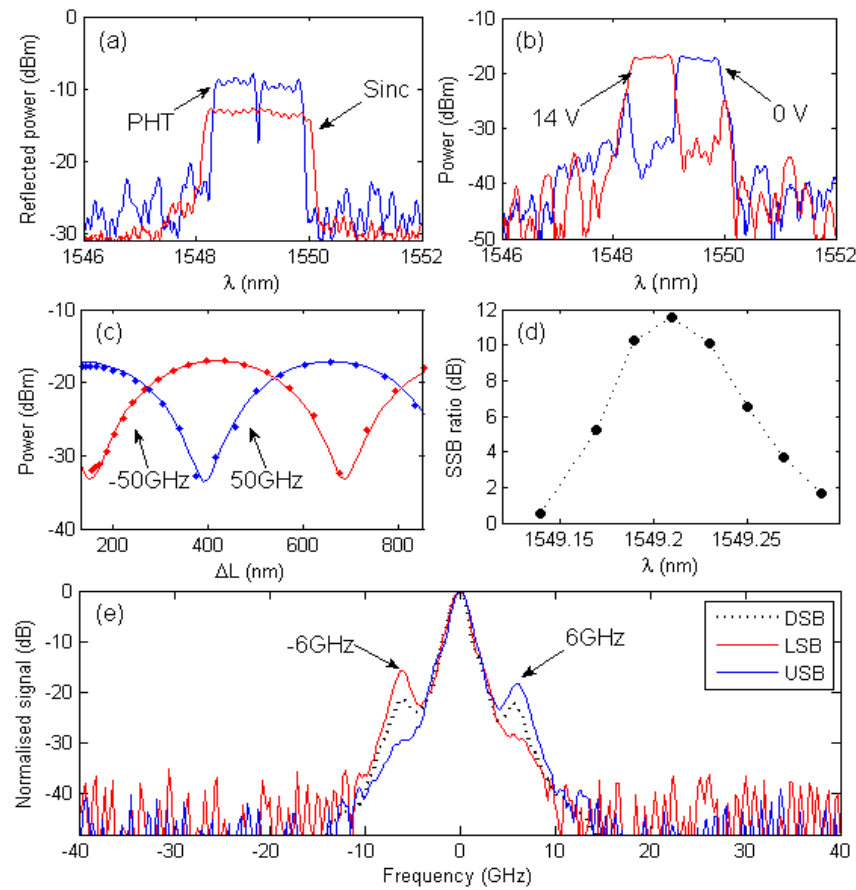

Fig. 4(a) Individual grating amplitude responses; (b) device output spectra with $0 \mathrm{~V}$ (blue) and $14 \mathrm{~V}$ (red) DC voltages; (c) modeled (line) and experimental data (dot) of output amplitude variations at $50 \mathrm{GHz}$ (blue) and $-50 \mathrm{GHz}$ (red) frequency interval; (d) the SSB suppression ratio with wavelength detuning at $6 \mathrm{GHz}$; (e) the input DSB signal (black dotted line), the output LSB signal (blue solid line) and the output USB signal (red solid line), the data is normalized for comparison.

The experimental performance of the fabricated device for microwave operation is investigated. Figure 4(a) shows the PHT (blue) and the sinc apodized grating (red) amplitude responses measured from ports C and D. Figure 4(b) shows the output spectra via thermal tuning at $0 \mathrm{~V}$ (blue) and $14 \mathrm{~V}$ (red), directly measured from port $\mathrm{B}$ of the device. Both figures (a) and (b) were measured using an OSA, 3dB coupler and an erbium doped fiber ASE source. Figure 4(c) shows the modeled data (solid line) and experimental data (dots) of the optical amplitude variation at $50 \mathrm{GHz}$ frequency interval (measured optically with the OSA) versus thermal tuning. Figure $4(\mathrm{~d})$ shows the SSB suppression ratio (defined as the ratio between the power of suppressive sideband and that of another sideband [5]) with wavelength detuning at $6 \mathrm{GHz}$. The maximum SSB suppression effect occurs when operating at the notch wavelength of the PHT spectrum, i.e. $1549.21 \mathrm{~nm}$.
The notch wavelength shift was obtained if the entire chip was heated up [13]. Figure 4(e) shows the input DSB signal at $6 \mathrm{GHz}$ (black dotted line), the lower sideband signal (LSB, blue solid line) and the upper sideband signal (USB, red solid line). The measurements show a $12 \mathrm{~dB}$ SSB suppression ratio for the filtered sideband. The modulation bandwidth of the data presented (at $6 \mathrm{GHz}$ ) is limited by the available characterization equipment and not the device. The operation bandwidth could be up to 100 $\mathrm{GHz}$ according to the data shown in Fig.4 (b). The total insertion loss of the actual device was $\sim 20 \mathrm{~dB}$ due to coupling loss, propagation loss in the $\mathrm{X}$ coupler and low grating reflectivity. This can be reduced substantially by improving the X-coupler losses and using stronger gratings designed appropriately by inverse scattering methods [14].

In conclusion, we have modeled and experimentally demonstrated a continually tunable integrated all-optical SSB filter based on a photonic Hilbert transformer. A $12 \mathrm{~dB}$ sideband suppression ratio at $6 \mathrm{GHz}$ was obtained and maximized by thermal tuning via a micro heater. In the future, removal of the micro heater and greater SSB suppression could be achieved by passive phase trimming and optimization of the $\mathrm{X}$-coupler and grating designs which would reduce response ripple.

This work was supported by Engineering and Physical Sciences Research Council (EPSRC), UK and China Scholarship Council.

\section{References}

1. S. L. Hahn, in The Transforms and Applications Handbook, 2nd ed., A.D. Poularikas, ed. (CRC Press, 2000).

2. Z. Li, W. Li, H. Chi, X. Zhang, and J. P. Yao, IEEE Photon. Technol. Lett., 23, 558 (2011).

3. M. H. Asghari and J. Azaña, Opt. Lett., 34, 334 (2009).

4. A. D. McAulay, Appl. Opt., 39, 2300 (2000).

5. K. Takano, N. Hanzawa, S. Tanji and K. Nakagawa, in Optical Fiber Communication and the National Fiber Optic Engineers Conference, OSA Technical Digest Seris (CD) (Optical Society of America, 2007), Paper JThA48.

6. H. Emami, N. Sarkhosh, L. A. Bui, and A. Mitchell, Opt. Express, 16, 13707 (2008)

7. X. Wang, M. Hanawa, K. Nakamura, and K. Nakagawa, in Proceedings of the 15th Asia-Pacific Conference on Communications (APCC, Shanghai, 2009).

8. T. H. Huang, X. Yi, and R. A. Minasian, Opt. Lett., 36, 4440 (2011).

9. G. D. Emmerson, S. P. Watts, C. B. Gawith, V. Albanis, M. Ibsen, R. B. Williams, and P.G. R. Smith, Electron. Lett, 38, 1531 (2002).

10. F. R. Adikan, J. C. Gates, C. B. Gawith, and P. G. R. Smith, Electron. Lett., 42, 1397 (2006).

11. C. Sima, J. C. Gates, H. L. Rogers, C. Holmes, M. N. Zervas, and P. G.R. Smith, In Proceedings of the 16th European Conference on Integrated Optics (ECIO 2012).

12. K. Okamoto, in Fundamentals of Optical Waveguides, (Academic Press, 2006).

13. C. Holmes, D. O. Kundys, J. C. Gates, C. B. E. Gawith and P. G. R. Smith, Electron. Lett., 45, 954 (2009).

14. R. Feced, M. N. Zervas, and M. A. Muriel, J. Quantum Electron., 35, 1105-1115 (1999). 


\section{References}

1.S. L. Hahn, "The Transforms and Applications Handbook," 2nd ed., A. D. Poularikas, ed. (CRC Press LLC, Boca Raton, Fla., 2000).

2. Z. Li, H. Chi, X. Zhang, and J. P. Yao, "Optical singlesideband modulation using a fiber-Bragg-grating-based optical Hilbert transformer," IEEE Photon. Technol. Lett., 23, 558-560 (2011).

3. M. H. Asghari and J. Azaña, "All-optical Hilbert transformer based on a single phase-shifted fiber Bragg grating: Design and analysis," Opt. Lett., 34, 334-336 (2009).

4. A. D. McAulay, "Hilbert transform and mirror-image optical correlators," Appl. Opt., 39, 2300-2309 (2000).

5. K. Takano, N. Hanzawa, S. Tanji and K. Nakagawa, "Experimental demonstration of optically phase-shiftd SSB modulation with Fiber-based optical Hilbert transformers," in Optical Fiber Communication and the National Fiber Optic Engineers Conference, Technical Digest (Optical Society of America, Anaheim, CA, 2007), Paper JThA48.

6. H. Emami, N. Sarkhosh, L. A. Bui, and A. Mitchell, "Amplitude independent RF instantaneous frequency measurement system using photonic Hilbert transform," Opt. Express, 16, 13707-13712 (2008) .

7. X. Wang, M. Hanawa, K. Nakamura, K. Takano and K. Nakagawa, "Sideband Suppression Characteristics of Optical SSB Generation Filter with Sampled FBG Based 4-taps Optical Hilbert Transformer," in Proceedings of the 15th Asia-Pacific Conference on Communications (APCC, 2009), pp. 622-625.

8. T. H. Huang, X. Yi, and R. A. Minasian "Microwave photonic quadrature filter based on an all-optical programmable Hilbert transformer", Opt. Lett., 36, 4440-4442 (2011).

9. G. D. Emmerson, S. P. Watts, C. B. Gawith, V. Albanis, M. Ibsen, R. B. Williams, and P. G. R. Smith "Fabrication of directly UV-written channel waveguides with simultaneously defined integral Bragg gratings", Electron. Lett, 38, 1531-1532 (2002).

10. F. R. M. Adikan, J. C. Gates, C. B. E. Gawith, and P. G. R. Smith, " Coupling ratio tuning of direct UV-written Xcouplers for cascaded power splitters in WDM networks," Electron. Lett., 42(24), 1397-1398 (2006).

11. C. Sima, J. C. Gates, H. L. Rogers, P. Mennea, C. Holmes, M. N. Zervas, and P. G.R. Smith, "Interferometric integrated planar Bragg filter for alloptical single-sideband suppression," the $16^{\text {th }}$ European Conference on Integrated Optics (ECIO 2012), Barcelona 18-20 April 2012

12. K. Okamoto, "Fundamentals of Optical Waveguides," (Academic Press, San Diego, 2006).

13. C. Holmes, D. O. Kundys, J. C. Gates, C. B. E. Gawith and P. G. R. Smith, " $150 \mathrm{GHz}$ of thermo-optic tuning in direct UV written silica-on-silicon planar Bragg grating," Electron. Lett., 45, 954-956 (2009).

14. R. Feced, M. N. Zervas, and M. A. Muriel, "An efficient inverse scattering algorithm for the design of nonuniform fiber Bragg gratings," J. Quantum Electron., 35, 1105-1115 (1999). 